\author{
Classification \\ Physics Abstracts \\ $7.500-8.860-9.130$
}

\title{
AMÉLIORATION DE L'EFFET DE SERRE DANS LES CONVERTISSEURS THERMIQUES DE L'ÉNERGIE SOLAIRE : TRAITEMENT ANTIREFLET DE LA COUVERTURE DE VERRE, PAR VOIE CHIMIQUE
}

\author{
M. PAPINI et F. PAPINI \\ Département d'Héliophysique, E. R. A. au C. N. R. S. n 538 , \\ Université de Provence, Centre de Saint Jérôme, \\ 13397 Marseille Cedex 4, France
}

(Reçu le 5 avril 1977, révisé le 18 juillet 1977, accepté le 5 septembre 1977)

\begin{abstract}
Résumé. - On expose ici une méthode de traitement par voie chimique de la couverture de verre d'un absorbeur solaire, permettant de diminuer les pertes par réflexion sur les deux dioptres verre-air. On obtient ainsi une augmentation du facteur de transmission, pour le spectre solaire, d'environ $6 \%$, cette propriété étant conservée lorsque l'angle d'incidence des rayons varie. Une application aux capteurs plans ainsi qu'aux capteurs à concentration est ensuite décrite.

Abstract. - A chemical treatment of the cover in a solar collector is presented which allows a reduction of losses due to the reflection on the two dioptres glass-air.

An increase of the transmission factor of about $6 \%$ is obtained for the solar spectra, this property being conserved when the incident rays angle varies.

An application to flat plate collectors and to focusing collectors is described.
\end{abstract}

1. Introduction. - La conversion thermique du rayonnement solaire conduit à utiliser des absorbeurs noirs ou sélectifs, selon les conditions d'emploi $[1,2,3]$. En effet, un fonctionnement idéal du convertisseur est obtenu lorsque les pertes thermiques sont négligeables vis-à-vis de la puissance captée. Si la température d'utilisation n'est pas suffisamment faible, cette condition peut alors être satisfaite, soit en diminuant le facteur d'émission de la surface absorbante, tout en lui conservant un facteur d'absorption proche de l'unité, soit en augmentant la concentration du rayonnement solaire incident. Le compromis est déterminé par l'étude de l'influence des propriétés optiques du foyer échangeur sur son bilan thermique.

Une méthode courante, permettant de diminuer le facteur d'émission apparent d'un convertisseur, consiste à disposer, devant la surface absorbante, une ou plusieurs convertures de verre, réalisant ainsi un effet de serre simple ou multiple. On obtient alors non seulement un affaiblissement des pertes infra-rouges, mais également une réduction de l'influence des agents atmosphériques, comme le vent, sur le bilan thermique de la surface absorbante elle-même; parallèlement, ceci s'accompagne, pour l'ensemble du système, d'une diminution du facteur d'absorption global du spectre solaire. Dans tous les systèmes de capteurs, munis d'un dispositif concentrateur du rayonnement ou non, la mise en place d'une vitre doit toujours être justifiée par l'accroissement du rendement de l'installation, ce qui conduit à envisager un compromis entre l'absorptivité et l'émissivité du dispositif.

Il est par conséquent intéressant d'effectuer un traitement de la couverture de verre de façon à diminuer son facteur de réflexion ou encore augmenter son facteur de transmission. Un tel traitement est obtenu par le dépôt d'une ou plusieurs couches minces sur le support de verre. Habituellement, celles-ci sont évaporées, dans une enceinte à vide, à partir d'un creuset chauffé contenant la substance massive à déposer. Cette technologie s'avère cependant d'un emploi délicat; nous avons donc testé un procédé de traitement du verre par voie chimique ne nécessitant pas d'appareillage spécifique $[4,5]$; il s'agit d'effectuer une attaque superficielle provoquant l'apparition d'une couche mince d'un matériau dont l'indice de réfraction est inférieur à celui du verre. Notons que cette attaque se fait sur toute la superficie, entraînant la présence du traitement sur ses deux faces principales. Nous verrons qu'il est possible de réaliser un gain non négligeable sur le rayonnement solaire transmis, ce qui nous permettra de montrer la validité de l'application de ce procédé dans le cas des capteurs plans sans concentration de l'énergie incidente. Une généralisation aux capteurs à concentration sera également traitée dans un dernier paragraphe. Voyons tout d'abord la description du procédé chimique proprement dit. 
2. Traitement antireflet. - L'échantillon à traiter est préalablement nettoyé puis trempé pendant vingt à trente secondes dans de l'acide fluorhydrique à $0,5 \%$ avant rinçage. Il est ensuite plongé dans un bain porté à la température de $45^{\circ} \mathrm{C}$ et préparé à partir d'acide hexafluorosilicique 1,4 M, saturé en silice, filtré, puis amené à une concentration $1,25 \mathrm{M}$; une très faible quantité d'une solution d'acide borique à $4 \%$ est alors ajoutée afin d'obtenir un minimum du facteur de réflexion de l'ensemble couche-verre le plus faible possible pour $0,5 \mu \mathrm{m}$, longueur d'onde correspondant au maximum du spectre d'émission du soleil. Il faut noter que la température du bain et la concentration en acide borique doivent être soigneusement contrôlées; cette dernière est choisie en fonction de la nature du verre utilisé. Dans le cas présent, pour du verre à vitre du commerce d'une épaisseur de $3 \mathrm{~mm}, 3$ à $4 \mathrm{~cm}^{3}$ de solution d'acide borique sont ajoutés à un litre de solution de traitement. On peut alors suivre, au cours du temps, l'évolution de la courbe de réponse spectrale $R=f(\lambda)$ et arrêter l'attaque au moment voulu. La figure 1 permet de rendre compte d'une telle évo-

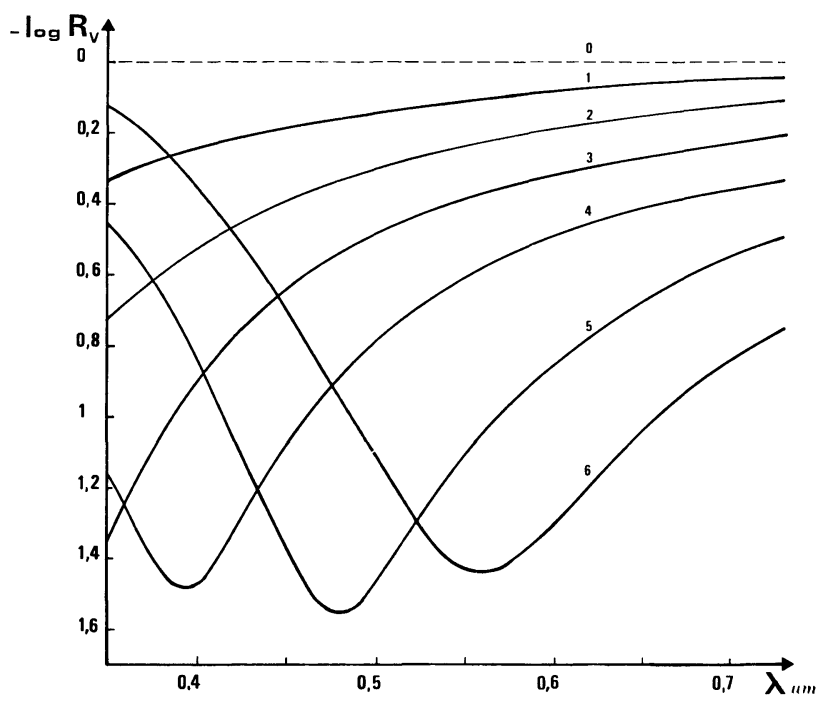

FIG. 1. - Evolution de la courbe de réponse spectrale du verre traité par rapport au verre non traité, en fonction du temps, lors du traitement chimique : courbe 0 : verre non traité ; courbe 1: $45 \mathrm{~min}$. ; courbe 2: $58 \mathrm{~min}$. ; courbe $3: 68 \mathrm{~min}$.; courbe $4: 75 \mathrm{~min}$. ; courbe $5: 86 \mathrm{~min}$. ; courbe $6: 97 \mathrm{~min}$.

[Evolution of the treated glass spectral response in comparison with the non-treated glass, versus the time, during the chemical treatment : curve $0:$ non treated glass; curve $1: 45 \mathrm{~min}$; curve $2: 58 \mathrm{~min}$. ; curve $3: 68 \mathrm{~min}$. ; curve $4: 75 \mathrm{~min}$. ; curve 5 : $86 \mathrm{~min}$. ; curve $6: 97 \mathrm{~min}$.]

lution. D'autre part, la figure 2 donne, pour un échantillon particulier, la courbe $R=f(\lambda)$, en incidence quasi normale, l'axe des abscisses étant gradué selon une échelle proportionnelle à un paramètre $k_{\mathrm{s}}$ défini par le rapport de la puissance solaire contenue dans la bande $|0-\lambda|$ à la puissance solaire totale ; le rayonnement incident étant assimilé à celui d'un corps noir porté à la température $T_{\mathrm{s}}=5880 \mathrm{~K}$, on a :

$$
k_{\mathrm{s}}=\frac{\int_{0}^{\lambda} M\left(\lambda, T_{\mathrm{s}}\right) \mathrm{d} \lambda}{\sigma T_{\mathrm{s}}^{4}}
$$

où $M\left(\lambda, T_{\mathrm{s}}\right)$ est l'émittance spectrale monochromatique d'un corps noir à la température $T_{\mathrm{s}}$ et $\sigma$ la constante de Stefan. Le paramètre $k_{\mathrm{s}}$ représente donc la proportion d'énergie solaire incluse dans la bande $|0-\lambda|$ et l'intégration de la courbe 2 permet d'obtenir directement le facteur de réflexion global pour le spectre solaire ; on obtient $R \simeq 3 \%$, le verre non traité ayant un facteur $R$ proche de $9 \%$.

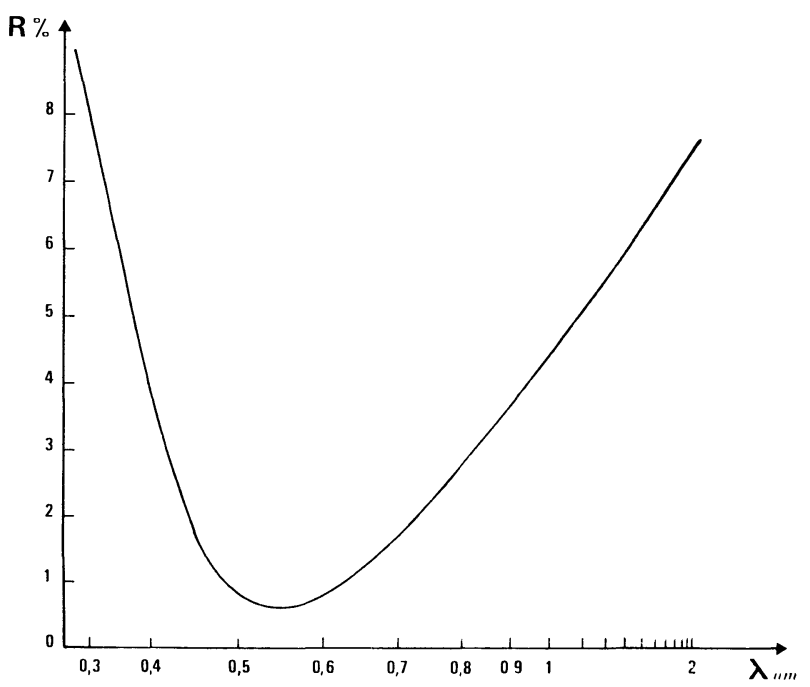

Fig. 2. - Facteur de réflexion du verre, en incidence quasi normale, en fonction de la longueur d'onde, après traitement chimique. L'axe des abscisses est gradué selon une échelle proportionnelle à $k$ s.

[Reflection factor of glass, versus the wavelength, after chemical treatment. The absciss axis is graduated with a scale proportional to $k_{\mathrm{s} \text {.] }}$

Afin de contrôler la fiabilité des résultats ainsi obtenus, nous avons porté les lames de verre antireflet à diverses températures pendant environ deux mille

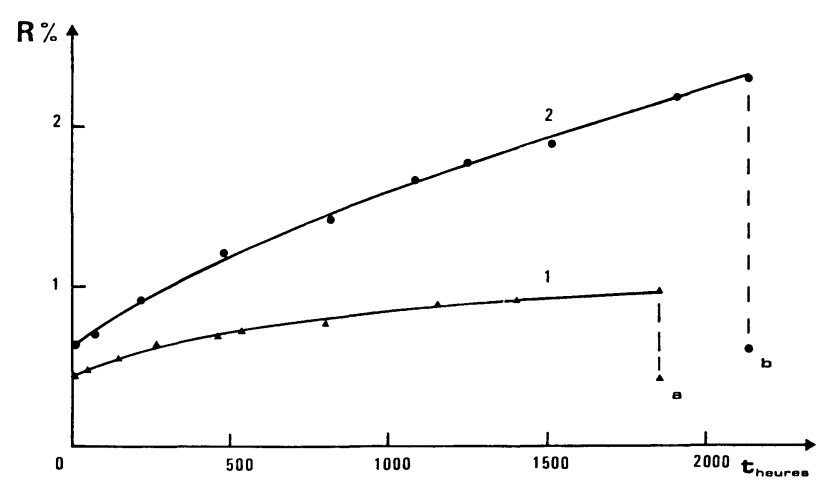

Fig. 3. - Variation du facteur de réflexion du verre traité en fonction du temps, pour la longueur d'onde $0,55 \mu \mathrm{m}$ : courbe 1 : $100^{\circ} \mathrm{C}$; courbe $2: 150^{\circ} \mathrm{C}$. Les points $\mathrm{a}$ et $\mathrm{b}$ sont obtenus après lavage de l'échantillon.

[Variation of the reflection factor of treated glass, versus the time, for the wavelength of $0.55 \mathrm{~m} \mu$ : curve $1: 100^{\circ} \mathrm{C}$; curve 2 : $150^{\circ} \mathrm{C}$. The points $\mathrm{a}$ and $\mathrm{b}$ are obtained after washing of the sample]. 
heures. Les variations de $R$, pour la longueur d'onde $0,55 \mu \mathrm{m}$, sont données sur la figure 3 . On note une faible augmentation du facteur de réflexion parallèlement à l'apparition en surface d'une couche très fine, difficilement caractérisable, qui disparaît après rinçage. Cette opération permet de retrouver des propriétés optiques proches de celles de l'échantillon fraîchement traité.

Avec ce procédé d'attaque par voie chimique, on peut donc obtenir un traitement antireflet de bonne qualité tant sur le plan des propriétés optiques que sur le plan de la fiabilité de ses performances. Malgré la nécessité d'un contrôle suffisamment précis de la concentration du bain utilisé, on dispose d'une méthode relativement facile à mettre en œuvre et qui permet d'éviter l'emploi de la technologie du vide.
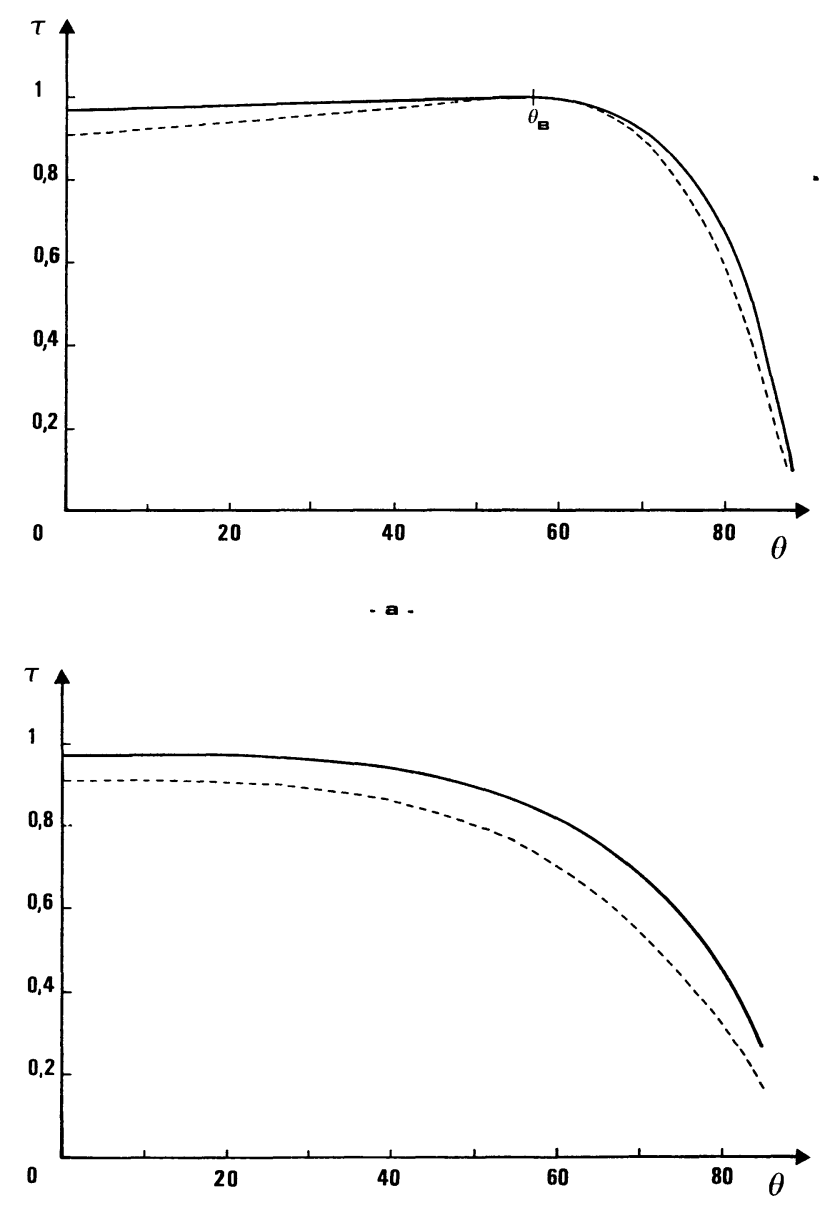

- b -

Fig. 4. - Variations du facteur de transmission en fonction de l'angle d'incidence $\theta$, en degrés, pour la longueur d'onde $0,47 \mu \mathrm{m}$; traits pleins : vitre traitée ; traits pointillés : vitre non traitée. a) Polarisation $P$ (champ électrique parallèle au plan d'incidence) On notera l'angle de Brewster $\theta_{\mathrm{B}}$ pour lequel $\tau=1$. b) Polarisation $S$ (champ électrique perpendiculaire au plan d'incidence).

[Variation of the transmission factor versus incident angle $\theta$ in degrees, for the wavelength of $0.47 \mathrm{~m} \mu$ : continuous lines ; treated glass; dotted lines : non-treated glass. a) Polarization $P$ (Electric field parallel to the incident plane). One will note the Brewster angle $\theta_{\mathrm{B}}$ for which $\tau=1$. b) Polarization $S$

(Electric field perpendicular to the incident plane).]
3. Application aux capteurs plans. - Dans le cas des capteurs plans, c'est-à-dire des convertisseurs dépourvus de système de poursuite automatique du soleil, il est nécessaire de vérifier que, pour toutes les valeurs de l'angle d'incidence du rayonnement, le traitement permet d'obtenir un facteur de transmission au moins égal à celui du verre non traité. Ceci est d'autant plus important que les radiations diffuses, sans direction privilégiée, jouent un grand rôle dans le fonctionnement de ce type de capteurs.

Ainsi, la figure 4 donne, pour les deux états de polarisation de la lumière à la longueur d'onde de $0,47 \mu \mathrm{m}$, les facteurs de transmission du verre traité et non traité, en fonction de l'angle d'incidence $\theta\left({ }^{1}\right)$.

En tenant compte de ces deux états de polarisation, on peut constater que l'accroissement du facteur de transmission, de l'ordre de $6 \%$, est conservé lorsque l'angle $\theta$ varie entre $0^{\circ}$ et $90^{\circ}$. On peut donc affirmer qu'au cours d'une journée, le gain relatif sur le rayonnement direct est constant quelle que soit la position du soleil ; par ailleurs le gain relatif sur le rayonnement diffusé par la voûte céleste et les couches nuageuses est également de $6 \%$.

Ainsi, le traitement par voie chimique de la couverture de verre semble bien adapté aux capteurs plans ; ceci à cause, d'une part, de la facilité de mise en œuvre du procédé et, d'autre part, de l'accroissement du facteur de transmission, pratiquement constant en fonction de l'angle d'incidence du rayonnement solaire. On peut alors, pour une température de fonctionnement donnée, espérer obtenir une augmentation du rendement instantané et journalier de l'ordre de $6 \%$. $\mathrm{Au}$ cours du paragraphe suivant, dans un cadre plus général où l'on admet en outre une concentration du rayonnement incident, nous allons définir les conditions d'emploi d'un effet de serre avec vitre traitée par rapport à celles correspondant à une couverture non traitée.

4. Généralisation. - Comme nous l'avons déjà dit en introduction, tout convertisseur thermique de l'énergie doit être conçu à partir de divers compromis et ceci, compte-tenu des conditions extérieures, comme en particulier la valeur de la puissance solaire incidente et la vitesse du vent. Que le dispositif de conversion soit muni d'un système de concentration du rayonnement ou non, il est important de savoir si l'adjonction d'une vitre apporte une amélioration au rendement. Nous allons montrer ici que le traitement antireflet que nous avons décrit apporte de sensibles modifications aux conditions d'emploi de l'effet de serre. Pour cela, nous effectuerons un calcul simple, permettant de comparer le rendement d'un absorbeur noir plan, dépourvu de couverture de verre, à celui d'un absorbeur équipé d'un effet de serre. Le capteur solaire sera

(1) Nous tenons à remercier Jacques Lafait et Josette Rivory, du Laboratoire d'Optique des Solides de l'Université de Paris VI, qui ont effectué ces mesures. 
caractérisé par son coefficient de concentration géométrique $C$, égal au rapport de la surface apparente de captation (miroirs) à la surface apparente d'absorption (foyer); dans le cas du capteur plan $C=1$. D'autre part, on désignera par $H$ (exprimée en $\mathrm{W} . \mathrm{m}^{-2}$ ) la puissance solaire incidente, rapportée à l'unité de surface de la pupille d'entrée de l'absorbeur. Ainsi, le produit $\mathrm{CH}$ est la puissance effectivement utilisable au niveau de l'entrée du système d'absorption; c'est une grandeur instantanée, pouvant être variable avec l'angle d'incidence du rayonnement solaire. Enfin, on imposera une égalité d'aire entre la surface absorbante et la vitre, et on considèrera que le facteur d'émission de cette dernière est égal à l'unité.

Nous allons ainsi écrire le bilan thermique d'une surface équipée ou non d'une couverture et soumise à un vent de vitesse $v$ variable. La température de fonctionnement du capteur est prise égale à $425 \mathrm{~K}$. On obtient alors, $P_{\mathrm{c}}$ représentant les pertes par convection naturelle dans l'espace vitre-absorbeur et $h_{\mathrm{v}}$ le coefficient de transfert de la chaleur dans l'environnement (par convection forcée ou naturelle) :

\subsection{SANS EFFET DE SERRE. -}

$$
\varphi_{\mathrm{u}}+\sigma T^{4}+h_{\mathrm{v}}\left(T-T_{\mathrm{a}}\right)=\mathrm{CH}+\sigma\left(T_{\mathrm{a}}-6\right)^{4} .
$$

La puissance correspondant au rayonnement du ciel est donnée ici par la formule de A. Whillier [6], $T_{\mathrm{a}}$ étant la température ambiante ; d'autre part, la puissance récupérable sous forme de chaleur, à l'aide d'un fluide caloporteur, est notée $\varphi_{\mathbf{u}}$.

4.2 AVEC EFFET DE SERRE. - au niveau de l'absorbeur à la température $T$ :

$$
\varphi_{\mathrm{u}}+\sigma T^{4}+P_{\mathrm{c}}=\tau C H+\sigma T_{\mathrm{v}}^{4}
$$

- au niveau de la vitre à la température $T_{\mathrm{v}}$ :

$$
\begin{array}{r}
2 \sigma T_{\mathrm{v}}^{4}+h_{\mathrm{v}}\left(T_{\mathrm{v}}-T_{\mathrm{a}}\right)=\sigma T^{4}+P_{\mathrm{c}}+\alpha^{\prime} \mathrm{CH}+ \\
+\sigma\left(T_{\mathrm{a}}-6\right)^{4}
\end{array}
$$

où $\alpha^{\prime}$ est le facteur d'absorption de la vitre pour le spectre solaire $\left(\alpha^{\prime} \simeq 5 \%\right)$ et $\tau$ son facteur de transmission.

On a pris pour les pertes par convection naturelle dans l'espace vitre-absorbeur : $P_{\mathrm{c}}=1,2\left(T-T_{\mathrm{v}}\right)^{1,25}$. Par ailleurs, le coefficient de transfert de la chaleur $h_{\mathrm{v}}$ dépend de la vitesse du vent $v$, de l'orientation et des dimensions des surfaces d'échange $[7,8]$; on a adopté, dans des conditions moyennes d'utilisation, la relation approchée suivante :

$$
\begin{gathered}
h_{\mathrm{v}}=5+3,8 v \\
\left(h_{\mathrm{v}} \text { est en } \mathrm{W} \cdot \mathrm{m}^{-2} \cdot \mathrm{K}^{-1} \text { et } v \text { en } \mathrm{m} \cdot \mathrm{s}^{-1}\right) .
\end{gathered}
$$

On peut alors évaluer la valeur $U$ de $\mathrm{CH}$ (en $\mathrm{W} . \mathrm{m}^{-2}$ ), en fonction de la vitesse du vent $v$, pour laquelle l'effet de serre permet d'obtenir un rendement égal à celui de la plaque absorbante dépourvue de couverture de verre. La courbe ainsi tracée conduit à la détermination de deux zones de fonctionnement situées de part et d'autre du graphe : l'une pour l'absorbeur sans effet de serre, l'autre pour l'absorbeur avec effet de serre. La figure 5 a été établie pour une couverture non traitée et une couverture traitée chimique-

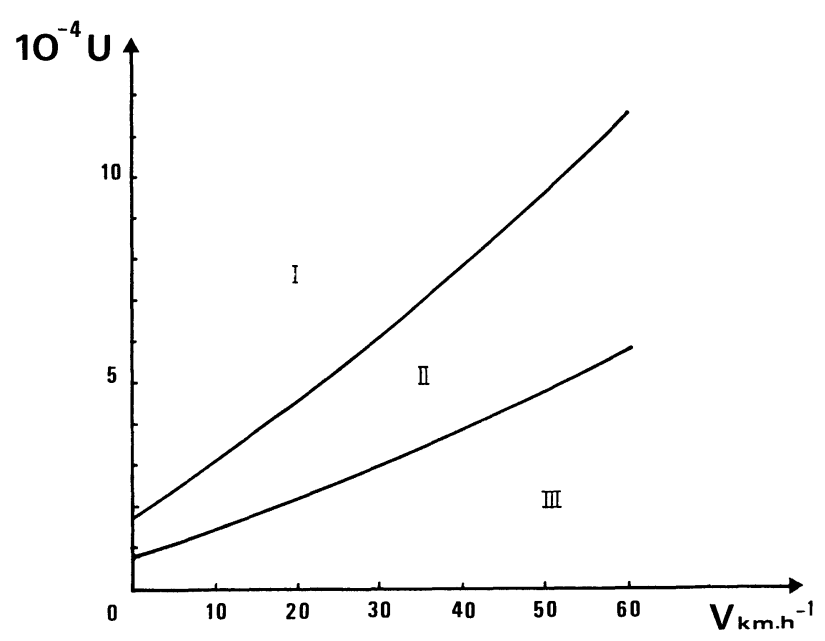

FIG. 5. - Courbes $U=f(v)$ pour du verre traité et non traité. La zone I correspond au domaine d'utilisation d'un corps noir ; la zone II à celui du corps noir ou de l'effet de serre avec verre traité ; la zone III à celui de l'effet de serre avec verre traité ou non traité.

[Curves $U=f(v)$ for a treated and non treated glass. The region I corresponds to the utilization of a blackbody; the region II to that of a blackbody or a greenhouse effect with treated glass; the region III to the greenhouse effect with treated or non-treated glass.]

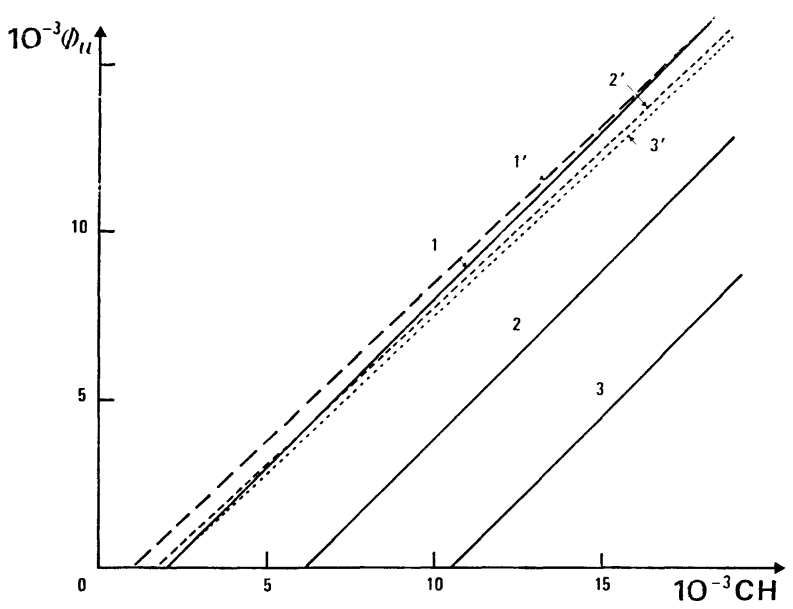

FIG. 6. - Comparaison des puissances récupérables en fonction de la puissance incidente pour un absorbeur porté à $425 \mathrm{~K}$ : - les courbes en traits pleins sont relatives au corps noir; - les courbes en traits pointillés sont relatives à l'absorbeur équipé d'une couverture de verre traité. 1 et $1^{\prime}: v=0 ; 2$ et $2^{\prime}$ : $v=30 \mathrm{~km} \cdot \mathrm{h}^{-1} ; 3$ et $3^{\prime}: v=60 \mathrm{~km} \cdot \mathrm{h}^{-1}$.

[Comparison with the useful powers versus the incident power for an absorber brought to $425 \mathrm{~K}:-$ the curves with continuous lines correspond to the blackbody; - the curves with dotted lines correspond to the absorber with a cover of treated glass. 1 and $1^{\prime}: v=0 ; 2$ and $2^{\prime}: v=30 \mathrm{~km} \cdot \mathrm{h}^{-1} ; 3$ and $3^{\prime}$ : $v=60 \mathrm{~km} \cdot \mathrm{h}^{-1}$.] 
ment. On y distingue donc trois zones de fonctionnement :

- zone I : absorbeur noir ;

- zone II : effet de serre avec verre traité ou absorbeur noir ;

— zone III : effet de serre avec verre traité ou non traité.

Afin de conserver au convertisseur solaire le maximum d'efficacité, on a donc toujours intérêt à travailler dans la zone adéquate, tenant compte ainsi des conditions d'éclairement et des pertes par convection forcée. La vitre assure une protection de l'échangeur lorsque ces paramètres évoluent au-delà d'un certain seuil ; si ce dernier n'est pas atteint, elle entraîne au contraire une perte optique provoquant une diminution du rendcment de l'appareil : elle devient donc inutile. Ainsi, sur la figure 6 , correspondant aux zones II et III, on constate que, grâce à la présence d'une couverture traitée, la puissance $\varphi_{\mathrm{u}}$ reste pratiquement constante.
On peut donc noter, la couverture de verre étant chimiquement traitée, que les conditions d'emploi de l'effet de serre sont notablement modifiées ; en particulier, le système antiperte peut être employé pour des valeurs plus faibles de la concentration $C$ et de la vitesse du vent $v$, ce qui contribue à augmenter son efficacité.

5. Conclusion. - Au moment où l'on cherche à mettre au point des surfaces sélectives chaudes à haut pouvoir absorbant par des efforts technologiques quelquefois importants, il est également nécessaire de supprimer les pertes par réflexion sur les dioptres de la couverture disposée en regard de cette surface. Pour cela, nous avons testé un procédé d'attaque par voie chimique permettant de réduire cette perte d'environ $6 \%$ et applicable aussi bien dans les capteurs plans sans concentration du rayonnement que dans les convertisseurs équipés d'un système focalisant.

\section{Bibliographie}

[1] Pasquetti, R. et Papini, F., Rev. Gen. Therm. 169 (1976) 11.

[2] Pasquetti, R. et Papini, F., Nouv. Rev. Opt. 7 (1976) 375.

[3] Pasquetti, R. et Papini, F., Solar Energy (à paraître).

[4] Nicoll, F. H., RCA Rev. 10 (1949) 440, 447.

[5] Thomsen, S. M., RCA Rev. 12 (1951) 143, 149.
[6] WhILLIER, A., Low temperature engineering applications of solar energy, New York, Ashrae, 1967.

[7] Mc Adams, W. H., Transmission de la chaleur, (Paris, Dunod) 1961.

[8] Kreith, F., Transmission de la chaleur et thermodynamique, (Paris, Masson) 1967. 